\title{
Influência do estado nutricional, distribuição da gordura corporal e força muscular na estabilometria de idosas
}

\author{
Influence of nutritional status, distribution \\ of body fat and muscle strength \\ on stabilometry of the elderly
}

Fabiano da Mota Silva SIQUEIRA ${ }^{1}$

Amandio Aristides Rihan GERALDES

\section{R E S U M O}

\section{Objetivo}

Investigar as associações entre diversas medidas indicadoras do estado nutricional, a força muscular e as diferentes variáveis estabilométricas em uma amostra de idosas.

\section{Métodos}

Neste estudo exploratório de corte transversal, 108 idosas tiveram suas variáveis estabilométricas avaliadas através de uma plataforma de força, em duas condições experimentais: olhos abertos e olhos fechados. Verificaram-se as associações entre as variáveis explicativas: idade, massa corporal, estatura, índice de massa corporal, circunferência de quadril, circunferência de cintura, relação cintura/quadril, percentual de gordura corporal, massa gorda, massa magra, área muscular de braço corrigida, força isométrica voluntária máxima e diversas variáveis estabilométricas: amplitude anteroposterior, deslocamento da oscilação e área elíptica da superfície, dentre outras.

\section{Resultados}

Com exceção da estatura, todas as variáveis explicativas associaram-se significativamente com as variáveis estabilométricas. A regressão múltipla (forward) revelou que a circunferência de cintura e a força isométrica voluntária máxima foram as variáveis que mais influenciaram as variáveis estabilométricas, entretanto sem diferenças significativas entre as condições olhos abertos e olhos fechados. O percentual de gordura parece não sobrecarregar o sistema de controle postural. Na condição olhos abertos, a variável estabilométrica desvio-

\footnotetext{
${ }^{1}$ Universidade Federal de Alagoas, Faculdade de Nutrição, Núcleo de Educação Física e Desportos. Campus AC Simões, Av. Lourival Melo Mota, s/n., Tabuleiro dos Martins, 57072-900, Maceió, AL, Brasil. Correspondência para/Correspondence to: AAR GERALDES. E-mail: <amandioargeraldes@gmail.com>.
} 
-padrão médio-lateral foi a mais influenciada pelas variáveis explicativas. A circunferência de cintura foi responsável por 8,8\% de toda variação do desvio-padrão médio-lateral e a força isométrica voluntária máxima contribuiu com $9,4 \%$ adicionais.

\section{Conclusão}

Os resultados sugerem que a distribuição centrípeta de gordura corporal associada à diminuição da força muscular são as principais responsáveis pelas alterações estabilométricas em idosas.

Palavras-chave: Composição corporal. Equilíbrio postural. Estado nutricional. Força muscular.

\section{A B S T R A C T}

\section{Objective}

To investigate the association and the relative contribution of different measurements used as indicators of nutritional status and muscle strength with different stabilometric variables in a sample of elderly.

\section{Methods}

In this cross-sectional study and exploratory, stabilometry with the use of force platform was performed with 108 elderly women selected in two experimental conditions: eyes open and eyes closed. It was found associations between explanatory variables: age, body mass, height, body mass index, hip circumference, waist circumference, waist to hip ratio, percentage of body fat, fat mass, lean body mass, corrected arm muscle area, maximum voluntary isometric strength and the stabilometric several variables: anteroposterior amplitude, cop displacement and elliptical surface area, among others.

\section{Results}

The explanatory variables associated significantly with the stabilometric variables, except height. Multiple regression analysis (forward) revealed that waist circumference and the maximum voluntary isometric strength were the variables that most influenced stabilometric variables without major significant differences between the conditions of eyes open and eyes closed. The \% body fat seems not to overload the system postural control sample of this study. The variable stabilometric mediolateral standard deviation (eyes open) was the most influenced by the explanatory variables. The waist circumference was responsible for $8.8 \%$ of all variation of mediolateral standard deviation and maximum voluntary isometric strenght contributed $9.4 \%$ additionally.

\section{Conclusion}

The regression analysis suggests that the centripetal distribution of body fat associated with reduced muscle strength are responsible, partly, for stabilometric changes in elderly women.

Keywords: Body composition. Postural balance. Nutritional status. Muscle strenght.

\section{N T R O D U Ç Ã O}

O envelhecimento é caracterizado por uma importante perda de funcionalidade ${ }^{1,2}$, e uma das consequências mais drásticas dessa perda é a queda. Sabe-se que durante o processo de envelhecimento há um aumento significativo no número de episódios de queda que pode ser atribuído ao declínio do desempenho do sistema de controle postural ${ }^{3}$.

O controle postural é mantido por vários sistemas: sensorial (somatossensorial, visual e vestibular), motor e um sistema de controle central de integração, que envolve complexas interações entre os vários sistemas neurais. Todos esses sistemas são afetados, de maneira independente, pelo envelhecimento ${ }^{3}$.

É de extrema relevância dispor de instrumentos que possam avaliar adequadamente o sistema de controle postural, principalmente, por sua relação com o risco de quedas. O mais utilizado e referido na literatura é a estabilometria com o uso da plataforma de força, que permite quantificar diversas variáveis derivadas do centro de pressão (do inglês center of pressure) ${ }^{3,4}$. Essas variáveis estabilométricas sofrem alterações significativas com o avançar da idade ${ }^{5}$, entretanto são muitos os fatores que podem contribuir para 
as alterações, como o medo de cair ${ }^{6}$, a interação medicamentosa ${ }^{7}$, algumas disfunções musculoesqueléticas ${ }^{8}$, doenças neurológicas ${ }^{3}$, dentre outras.

Além dos fatores extrínsecos e/ou patológicos que influenciam negativamente o controle postural e elevam os riscos de quedas, as alterações da morfologia e/ou da composição corporal podem ser consideradas possíveis fatores intrínsecos de instabilidade postural durante o processo de envelhecimento ${ }^{9,10}$. Nessa direção, mais recentemente, medidas e índices como a porcentagem de gordura corporal|11-13, o Índice de Massa Corporal (IMC) ${ }^{11,13}$, a massa muscular esquelética $^{13,14}$, a razão cintura/quadril ${ }^{11}$, a circunferência de cintura ${ }^{11}$, a circunferência de quadril ${ }^{11}$ e a força muscular ${ }^{14}$ vêm chamando a atenção dos pesquisadores pelo seu potencial de influenciar negativamente o controle postural dos sujeitos.

Durante o processo de envelhecimento, são verificadas alterações importantes na morfologia e na composição corporal. Verifica-se, por exemplo, a diminuição da massa corporal magra, principalmente da massa muscular esquelética, diminuição da força muscular, aumento progressivo e redistribuição da gordura corporal e acúmulo na região abdominal (distribuição centrípeta da gordura corporal) e nos quadris, que na mulher persiste até os 75 anos $^{10}$. De forma geral, são notáveis as prevalências de alterações do estado nutricional na população idosa, contudo não foi estabelecido se essas alterações influenciam as variáveis estabilométricas.

A prevalência de sobrepeso e de obesidade dentre as idosas é marcante e vem sendo associada às alterações do equilíbrio em alguns estudos ${ }^{13,15,16}$. Ademais, tem-se sugerido que o excesso de massa corporal, em associação com a distribuição centrípeta da gordura corporal, possa alterar o equilíbrio (tanto estático quanto dinâmico), podendo, portanto, modificar a localização do centro de massa corporal, levando à instabilidade do controle postural ${ }^{12}$.
Apesar de reconhecida a influência do excesso de peso e de adiposidade bem como o tipo de distribuição da gordura central ou periférica sobre a instabilidade postural, em sujeitos classificados com magreza excessiva ou baixo peso também foram observados alterações de equilíbrio e risco de quedas ${ }^{17,18}$. Alguns estudos vão além, observando que, em idosos, o aumento da massa corporal pode reduzir o risco de cair ${ }^{19}$.

Sabe-se também que a redução da força muscular e da massa muscular tem sido citada como importante fator de risco para limitações funcionais, representando um aspecto crítico para a alteração do equilíbrio postural do idoso 17,20,21. Há uma forte correlação positiva entre a massa muscular e a força muscular, entretanto existem evidências que indicam que as alterações quantitativas na massa muscular (hipotrofia) não são suficientes para explicar a perda de força associada ao envelhecimento ${ }^{22}$. Apesar de a perda de força muscular poder prejudicar o controle postural e aumentar o risco de quedas, a influência da diminuição da massa muscular sobre esses mecanismos não está totalmente esclarecida9,21.

Quanto à relação entre as variáveis antropométricas e o controle postural, um estudo recente ${ }^{14}$ comparou indivíduos eutróficos com obesos e grandes obesos (idade média de 40 anos) e identificou que, nos sujeitos com excesso de peso, a perda de peso é mais eficiente para melhorar o controle postural do que aumentar ou mesmo manter a força muscular.

Demonstra-se, portanto, que o exame da literatura revela-se controverso quando se tenta estabelecer se as alterações de equilíbrio, principalmente quando avaliadas pela estabilometria, estão associadas à massa corporal, à quantidade de massa ou força muscular, bem como à adiposidade e sua distribuição. Além do mais, ainda não estão totalmente esclarecidas as associações entre as variáveis estabilométricas e essas variáveis, especialmente entre a população idosa para a qual o risco de queda, além de ser mais elevado, representa um dos mais importantes preditores de morbimortalidade? 
Devido ao exposto, o objetivo do presente estudo exploratório foi investigar as associações entre diversas medidas estabilométricas com três diferentes variáveis utilizadas como indicadoras do estado nutricional (IMC, porcentagem de gordura corporal e aérea muscular de braço corrigida), tipo de distribuição de gordura corporal e força muscular em uma amostra de idosas. Adicionalmente, verificou-se a contribuição relativa das variáveis antropométricas e da força muscular para o desempenho de diferentes variáveis estabilométricas.

\section{M É T O D O S}

Para compor a amostra deste estudo de corte transversal e de caráter exploratório, foram selecionadas, de maneira conveniente, 108 adultas idosas, fisicamente independentes, engajadas em atividades de lazer ofertadas por cinco instituições de terceira idade do município de Maceió (AL).

Para participar da amostra, o sujeito deveria ser do sexo feminino; não estar realizando, há pelo menos seis meses, treinamento objetivando o aumento da força muscular; ser voluntário e assinar o Termo de Consentimento Livre e Esclarecido (TCLE).

Seriam excluídos da amostra os sujeitos que apresentassem: desordens neurológicas e cognitivas autorreferidas (exemplo: Parkinson, acidente vascular encefálico), tivessem realizado cirurgia da coluna vertebral e/ou membros inferiores e aqueles portadores de condições clínicas que pudessem impedir a realização das atividades propostas (exemplos: cardiopatias agudas, doenças pulmonares, hipertensão não controlada, amputações de membros, dentre outras), alterações visuais graves e alterações brandas sem correção; e quaisquer alterações que impedissem o registro das medidas antropométricas, de força muscular e/ou estabilométricas.

O estudo foi realizado em conformidade com os padrões éticos estabelecidos na Declaração de Helsinque e aprovado pelo Comitê de
Ética em Pesquisa da Universidade Federal de Alagoas, sob o Processo de $n^{\circ}$ 000741/2011-01.

Os sujeitos da amostra leram e assinaram o TCLE, e todos os procedimentos foram realizados por pesquisadores devidamente treinados para as funções, com instrumentos previamente calibrados e certificados pelos órgãos competentes.

As coletas foram realizadas nas dependências das próprias instituições de terceira idade em duas etapas: no primeiro encontro, os sujeitos foram informados e esclarecidos sobre os objetivos da pesquisa, os procedimentos e medidas a serem realizados (medidas antropométricas e de Força Isométrica Voluntária Máxima [FIVM] e avaliação estabilométrica), e após a leitura as voluntárias assinaram o TCLE. Ainda no primeiro encontro, realizou-se a aplicação do questionário para coleta das informações sociodemográficas e dos dados gerais de saúde. Após preenchimento e entrega dos questionários, foram agendados os encontros para a realização das medidas antropométricas, da FIVM e da estabilometria.

Todos os sujeitos foram instruídos quanto ao vestuário (traje de banho ou menor quantidade de roupa possível) a ser utilizado no dia da coleta das variáveis antropométricas. Para a avaliação estabilométrica e FIVM, as voluntárias foram orientadas a não fazerem uso de qualquer bebida estimulante (café, chá, energético ou álcool) ou tabaco, durante as 24 horas que antecederem a realização das medidas.

\section{Avaliação antropométrica e medidas de estado nutricional}

Foram registradas as medidas de circunferência de braço, cintura, quadril e coxa, dobra cutânea bicipital, tricipital e coxa, além da massa corporal e estatura. Para as medidas da massa corporal, utilizou-se balança digital portátil (Plenna ${ }^{\circledR}$, Modelo MEA-07400, Measurement Specialities Inc., Fremont, Califórnia, Estados Unidos), e, para a aferição da estatura, um esta- 
diômetro de parede portátil (Seca ${ }^{\circledR}$, Baystate Scale e Systems, Burlington, Massachusetts, Estados Unidos). Para a mensuração das circunferências, utilizou-se uma trena antropométrica metálica, inextensível, com precisão de 0,1 cm (Sanny ${ }^{\circledR}$, São Bernardo do Campo, São Paulo). As medidas das pregas cutâneas foram realizadas com um adipômetro com precisão de 0,1 mm (Lange ${ }^{\circledR}$, Beta Technology Incorporated, Cambridge, Maryland, Estados Unidos). Todas as medidas antropométricas foram realizadas de acordo com os protocolos recomendados por Lohman et al. ${ }^{23}$.

O índice de massa corporal foi calculado por meio da razão entre a massa corporal e o quadrado da estatura $\left(\mathrm{IMC}=\mathrm{Mc}(\mathrm{kg}) / \mathrm{Est}^{2}(\mathrm{~m})\right)$. A massa corporal e a estatura foram incluídas na análise: a primeira pela forte associação ao IMC e gordura corporal, e a estatura por ser frequentemente relacionada às alterações do centro de pressão ${ }^{24}$.

Para o cálculo da área muscular de braço corrigida, utilizou-se a equação para mulheres proposta por Frisancho ${ }^{25}$, onde $A M B c$ é a Área Muscular de Braço corrigida, CB é a Circunferência de Braço e DCT é a Dobra Cutânea Tricipital, ambas expressas em centímetros (cm): $\mathrm{AMBC}$ $\left(\mathrm{cm}^{2}\right)=[C B(\mathrm{~cm})-\pi \times \text { DCT }(\mathrm{cm}) / 10]^{2}-6,54 \pi$.

A área muscular de braço corrigida é utilizada na avaliação da reserva de tecido muscular corrigindo a área óssea e tem sido descrita como a medida teórica mais correta para a avaliação indireta da massa muscular em idosos, além de permitir distinguir adequadamente indivíduos eutróficos e desnutridos ${ }^{25}$.

A Relação de Cintura/Quadril (RCQ), um dos indicadores mais utilizados no diagnóstico de obesidade central, foi calculada pela razão entre as medidas da Circunferência de Cintura (CC) e Circunferência de Quadril (CQ) em centímetros $(\mathrm{cm})$, pela fórmula $\mathrm{RCQ}=\mathrm{CC} / \mathrm{CQ}$. Tanto a relação de cintura/quadril quanto as circunferências de cintura e quadril isoladamente foram incluídas na análise visto poderem estar associadas às alterações da estabilidade postural11,24.
Para o cálculo da porcentagem de Gordura Corporal (\%GC), utilizou-se a equação proposta por Oliveira \& Geraldes ${ }^{26}$, especialmente construída para esse tipo de população: \%GC=15,329 $+1,044$ (IMC) -1,055 (Cant) + 0,282 (CQ) + 0,164 (DCCX) - 0,262 (CCox), onde Cant: representa a Circunferência de antebraço expressa em cm, CQ: a Circunferência de Quadril, CCox: a Circunferência de Coxa, e DCCx: a Dobra Cutânea de Coxa, expressa em $\mathrm{mm}$.

\section{Avaliação da força isométrica voluntária máxima}

Neste estudo, escolheu-se a força isométrica voluntária máxima dos extensores de joelho como medida da força muscular devido às suas correlações com a força global ${ }^{23}$ e pelo fato de esta medida ser utilizada como preditora de quedas em idosos ${ }^{21}$.

A força isométrica voluntária máxima dos músculos extensores do joelho foi avaliada com auxílio de um transdutor de força, tipo célula de força, com capacidade de 2000 N (EMG System do Brasil Ltda., São José dos Campos, São Paulo), adaptado a um aparelho de Bonnet modificado, sem os braços de resistência. Para análise dos sinais, utilizou-se o software MATLAB (MathWorks Inc., Natick, Massachusetts, Estados Unidos) versão 7.10 (autorun). A taxa de amostragem foi de $2000 \mathrm{~Hz}$ e dotada de um filtro passa-faixa de 23 $\mathrm{Hz}$. Para a realização da medida da FIVM de extensores do joelho, a voluntária deveria estar sentada, com as costas apoiadas no encosto da cadeira de Bonnet adaptada, joelhos fletidos a $90^{\circ}$ (medidos com auxílio de um goniômetro Universal), tendo suas coxas presas à cadeira com faixas de velcro, visando a estabilizar sua postura ${ }^{27}$. Durante a realização da medida, uma correia de couro colocada em volta do tornozelo do membro testado era acoplada a uma célula de carga por meio de uma corrente que, por sua vez, era fixada à base da cadeira. 
Para essa medida, eram realizadas três contrações voluntárias isométricas máximas do quadríceps femoral bilateralmente no movimento de extensão do joelho. Cada contração deveria ser mantida durante cinco segundos. Como medida final, registrou-se o maior valor de força obtido dentre as três tentativas.

\section{Avaliação estabilométrica}

A estabilometria foi realizada com auxílio de uma plataforma de força (EMG System do Brasil Ltda., São José dos Campos, São Paulo) com dimensões de $50 \mathrm{~cm}$ de largura por $50 \mathrm{~cm}$ de comprimento, composta por quatro células de força. O processamento das variáveis estabilométricas foi realizado pelo software MATLAB 7.10 (autorun). A frequência de aquisição utilizada foi de $100 \mathrm{~Hz}$; com o objetivo de minimizar os ruídos, utilizou-se um filtro passa-baixa de $23 \mathrm{~Hz}$.

Para a realização da avaliação estabilométrica, a plataforma foi posicionada em superfície plana em frente a uma parede branca com um alvo disposto à altura da glabela da voluntária avaliada, a um metro de distância. O centro de pressão foi registrado em duas diferentes condições: olhos abertos e olhos fechados.

Foi solicitado a cada avaliada que permanecesse em bipedestação sobre a plataforma com os naviculares posicionados no centro da plataforma a uma distância de dois centímetros e um ângulo de abertura anterior de trinta graus. $\mathrm{Na}$ primeira medida da condição olhos abertos, os contornos dos pés foram marcados para que o sujeito retornasse à mesma posição após o período de repouso.

Durante a avaliação estabilométrica, os membros superiores dos sujeitos deveriam permanecer ao longo do tronco, e o olhar (na condição olhos abertos) fixado na marca posicionada na parede. Os sujeitos foram orientados a permanecerem imóveis o máximo possível, por 60 segundos, em cada condição para registro do centro de pressão com um intervalo de dois minutos entre cada registro. Conforme as orientações de Lafond et al. ${ }^{28}$, as medidas foram reali- zadas em duplicata e utilizaram-se as médias aritméticas de cada análise do centro de pressão.

Embora sejam várias as possibilidades de medidas obtidas através da estabilometria, um número limitado, composto pelas medidas consideradas variáveis fundamentais para a análise do centro de pressão, tem sido mais utilizado nas pesquisas ${ }^{4}$. Portanto, para o presente estudo, as seguintes medidas foram analisadas: 1) Área elíptica do estatocinesiograma: superfície de confiança que contém 95\% das posições amostradas do centro de pressão; 2) Desvio-padrão anteroposterior e médio-lateral: que comumente indica para altos valores do desvio-padrão grande instabilidade postural ${ }^{29}$; 3) Deslocamento da oscilação total: Tamanho ou comprimento da trajetória do centro de pressão sobre a base de suporte; 4) Velocidade média anteroposterior e médio-lateral; amplitude anteroposterior e médio-lateral, e 5) Frequência média anteroposterior e médio-lateral.

\section{Análise estatística}

O Teste de Kolmogorov-Smirnov foi utilizado para verificar se os dados apresentavam distribuição normal. Para a caracterização da amostra, utilizaram-se os valores da média aritmética, o desvio-padrão e a amplitude. Os coeficientes de correlação foram computados para determinar as associações simples entre as variáveis explicativas (idade, massa corporal, estatura, IMC, circunferência de cintura, razão cintura/quadril, porcentagem de gordura corporal, massa gorda, massa magra, área muscular de braço corrigida e FIVM) e as variáveis respostas (variáveis estabilométricas).

A idade e a estatura foram incluídas na análise de regressão porque têm sido apontadas como variáveis responsáveis por importantes alterações do centro de pressão em alguns estu$\operatorname{dos}^{11,24,30}$. No caso de variáveis com distribuição não normal, realizou-se a transformação logarítmica neperiana das variáveis não normais.

A análise de regressão múltipla (forward) foi utilizada para determinar quais dentre as 
variáveis explicativas influenciavam as variáveis estabilométricas. Antes da construção dos modelos de equações, realizou-se a verificação da colinearidade ou multicolinearidade entre as variáveis explicativas. Para a escolha do modelo de regressão, respeitaram-se os pressupostos de normalidade, linearidade, colinearidade e homocedasticidade. O peso matemático de cada variável explicativa na equação ( $\beta$ ) bem como seu teste $t$ foram examinados e apresentados.
Tratando-se de um estudo exploratório, o modelo final de regressão escolhido para cada variável dependente (estabilométrica) deve conter o maior número de variáveis explicativas, respeitando-se os pressupostos apresentados anteriormente, bem como valores de $t$ e teste $\mathrm{F}$ com $p<0,05$. Em caso de violação do pressuposto da colinearidade, a variável com maior correlação linear com a variável estabilométrica era incluída na análise de regressão múltipla. Todos os resultados foram considerados significantes para um

Tabela 1. Características das voluntárias incluindo as variáveis idade, medidas de estado nutricional, variáveis antropométricas e estabilométricas nas condições com olhos abertos e olhos fechados (n=108). Maceió (AL), 2012

\begin{tabular}{|c|c|c|c|}
\hline Variáveis & M & DP & Amplitude \\
\hline Idade (anos) & 70,10 & 6,29 & $60,00-87,00$ \\
\hline Massa corporal (kg) & 64,63 & 12,00 & $41,90-99,90$ \\
\hline Estatura $(m)$ & 1,51 & 0,06 & $1,38-\quad 1,64$ \\
\hline Índice de massa corporal (kg/cm²) & 28,34 & 5,02 & $18,14-45,52$ \\
\hline Circunferência de cintura (cm) & 90,59 & 11,56 & $37,00-123,00$ \\
\hline Circunferência de quadril (cm) & 102,87 & 10,01 & $66,30-139,00$ \\
\hline Relação cintura/quadril & 0,89 & 0,11 & $0,74-\quad 1,79$ \\
\hline Área muscular de braço corrigida $\left(\mathrm{cm}^{2}\right)$ & 32,44 & 12,00 & $11,04-80,49$ \\
\hline Porcentagem de gordura corporal & 40,82 & 6,17 & $16,69-59,01$ \\
\hline Massa magra $(\mathrm{kg})$ & 37,68 & 4,47 & $28,40-50,16$ \\
\hline Massa gorda $(\mathrm{kg})$ & 26,95 & 8,82 & $6,99-57,60$ \\
\hline \multirow[t]{2}{*}{ Força muscular máxima isométrica (kg.f) } & 22,27 & 5,78 & $9,00-35,08$ \\
\hline & Estabilomet & & \\
\hline \multicolumn{4}{|l|}{ Olhos abertos } \\
\hline Desvio-padrão anteroposterior (cm) & 0,41 & 0,13 & $0,22-\quad 1,22$ \\
\hline Desvio-padrão médio-lateral (cm) & 0,34 & 0,12 & $0,17-\quad 0,96$ \\
\hline Deslocamento da oscilação total (cm) & 83,94 & 19,81 & $53,84-166,19$ \\
\hline Amplitude anteroposterior $(\mathrm{cm})$ & 2,33 & 0,66 & $1,41-\quad 5,07$ \\
\hline Amplitude médio-lateral $(\mathrm{cm})$ & 1,92 & 0,66 & $0,95-\quad 4,88$ \\
\hline Área elíptica do estatocinesiograma $\left(\mathrm{cm}^{2}\right)$ & 2,78 & 2,27 & $0,74-21,16$ \\
\hline Velocidade média anteroposterior $(\mathrm{cm} / \mathrm{s})$ & 0,98 & 0,26 & $0,62-\quad 2,17$ \\
\hline Velocidade média médio-lateral (cm/s) & 0,80 & 0,20 & $0,56-\quad 1,71$ \\
\hline Frequência média anteroposterior ( $\mathrm{Hz})$ & 0,35 & 0,12 & $0,12-\quad 0,74$ \\
\hline Frequência média médio-lateral (Hz) & 0,34 & 0,11 & $0,15-\quad 0,71$ \\
\hline \multicolumn{4}{|l|}{ Olhos fechados } \\
\hline Desvio-padrão anteroposterior $(\mathrm{cm})$ & 0,47 & 0,19 & $0,23-\quad 1,69$ \\
\hline Desvio-padrão médio-lateral (cm) & 0,39 & 0,15 & $0,18-\quad 1,00$ \\
\hline Deslocamento da oscilação total (cm) & 102,32 & 36,67 & $56,12-281,01$ \\
\hline Amplitude anteroposterior $(\mathrm{cm})$ & 2,73 & 1,02 & $1,38-\quad 8,83$ \\
\hline Amplitude mediolateral $(\mathrm{cm})$ & 2,22 & 0,80 & $1,13-\quad 5,29$ \\
\hline Área elíptica do estatocinesiograma $\left(\mathrm{cm}^{2}\right)$ & 3,70 & 3,29 & $0,98-28,80$ \\
\hline Velocidade média anteroposterior $(\mathrm{cm} / \mathrm{s})$ & 1,23 & 0,49 & $0,60-\quad 3,40$ \\
\hline Velocidade média médio-lateral (cm/s) & 0,94 & 0,32 & $0,53-\quad 2,84$ \\
\hline Frequência média anteroposterior ( $\mathrm{Hz})$ & 0,38 & 0,13 & $0,17-\quad 0,83$ \\
\hline Frequência média médio-lateral (Hz) & 0,36 & 0,12 & $0,15-\quad 0,86$ \\
\hline
\end{tabular}

Nota: M: Média; DP: Desvio-Padrão. 
nível de $5 \%(p<0,05)$. O software estatístico Predictive Analytics Software (PASW, IBM Corporation, Armonk, Nova York, Estados Unidos), versão 18.0 foi utilizado para todas as análises.

\section{RES U L T A D O S}

As características físicas (idade, medidas antropométricas, medidas de estado nutricional e FIVM) bem como os valores médios de todas as variáveis estabilométricas nas condições olhos abertos e fechados dos sujeitos estão dispostos na Tabela 1.

Nas Tabelas 2 e 3 encontram-se os coeficientes de correlação linear das associações entre as variáveis explicativas e as variáveis estabilométricas nas situações de olhos abertos e fechados, respectivamente.

Tabela 2. Coeficientes de correlação das variáveis explicativas e variáveis estabilométricas na condição olhos abertos das idosas. Maceió (AL), 2012.

\begin{tabular}{|c|c|c|c|c|c|c|c|c|c|c|c|c|}
\hline Variável & Idade & $M C$ & Est & IMC & $C C$ & $C Q$ & $\mathrm{RCQ}$ & $\% G C$ & MM & MG & $A M B C$ & FIVM \\
\hline DPAP & $0,04^{2}$ & $0,20^{2^{*}}$ & $0,07^{2}$ & $0,16^{2}$ & $0,21^{2^{*}}$ & $0,16^{2}$ & $0,20^{22^{*}}$ & $0,07^{2}$ & $0,24^{2^{*}}$ & $0,17^{2}$ & $0,09^{2}$ & $-0,10^{2}$ \\
\hline DPML & $0,20^{2^{*}}$ & $0,21^{2^{*}}$ & $0,07^{2}$ & $0,20^{2^{*}}$ & $0,272^{* *}$ & $0,23^{2^{*}}$ & $0,15^{2}$ & $0,12^{2}$ & $0,20^{2^{*}}$ & $0,192^{*}$ & $0,192^{*}$ & $-0,23^{2^{*}}$ \\
\hline DOT & $0,28^{2^{* *}}$ & $-0,05^{2}$ & $-0,04^{2}$ & $0,01^{2}$ & $0,09^{2}$ & $0,09^{2}$ & $-0,01^{2}$ & $0,10^{2}$ & $-0,18^{2}$ & $0,01^{2}$ & $-0,06^{2}$ & $-0,01^{2}$ \\
\hline AmpAP & $0,05^{2}$ & $0,22^{2^{*}}$ & $0,05^{2}$ & $0,20^{2^{*}}$ & $0,25^{2^{* *}}$ & $0,22^{2^{*}}$ & $0,19^{2^{*}}$ & $0,13^{2}$ & $0,22^{2^{*}}$ & $0,21^{2^{*}}$ & $0,10^{2}$ & $-0,04^{2}$ \\
\hline AmpML & $0,262^{* *}$ & $0,25^{2^{* *}}$ & $0,08^{2}$ & $0,24^{2^{*}}$ & $0,30^{2^{* *}}$ & $0,272^{* *}$ & $0,14^{2}$ & $0,16^{2}$ & $0,22^{2^{*}}$ & $0,23^{2^{*}}$ & $0,18^{2}$ & $-0,192^{*}$ \\
\hline AEE & $0,17^{2}$ & $0,23^{2^{*}}$ & $0,08^{2}$ & $0,20^{2^{*}}$ & $0,26^{2^{* *}}$ & $0,212^{*}$ & $0,192^{*}$ & $0,11^{2}$ & $0,23^{2^{*}}$ & $0,20^{2^{*}}$ & $0,15^{2}$ & $-0,202^{*}$ \\
\hline VelAP & $0,242^{* *}$ & $0,03^{2}$ & $-0,05^{2}$ & $0,06^{2}$ & $0,15^{2}$ & $0,18^{2^{*}}$ & $0,01^{2}$ & $0,20^{2^{*}}$ & $-0,14^{2}$ & $0,11^{2}$ & $-0,02^{2}$ & $-0,02^{2}$ \\
\hline VelML & $0,262^{*}$ & $-0,05^{1}$ & $-0,06^{1}$ & $-0,04^{1}$ & $0,06^{1}$ & $-0,02^{2}$ & $0,02^{2}$ & $0,05^{1}$ & $-0,202^{2^{*}}$ & $-0,08^{2}$ & $-0,11^{2}$ & $-0,08^{1}$ \\
\hline FMAP & $0,05^{2}$ & $-0,02^{1}$ & $-0,13^{1}$ & $0,03^{1}$ & $0,04^{1}$ & $0,05^{2}$ & $-0,03^{2}$ & $0,18^{1}$ & $-0,20^{2^{*}}$ & $0,03^{2}$ & $-0,03^{2}$ & $0,06^{1}$ \\
\hline FMML & $-0,09^{2}$ & $-0,09^{2}$ & $-0,02^{2}$ & $-0,05^{2}$ & $-0,06^{2}$ & $-0,09^{2}$ & $-0,03^{2}$ & $0,03^{2}$ & $-0,13^{2}$ & $-0,04^{2}$ & $-0,06^{2}$ & $0,34^{2^{* \star}}$ \\
\hline
\end{tabular}

Nota: ${ }^{* *} 0,05>p>0,01 ;{ }^{* *} p<0,01 ;{ }^{1}$ Os valores apresentados são coeficientes de correlação de Pearson para os dados com distribuição normal; ${ }^{2}$ Spearman para os com distribuição não normal.

MC: Massa Corporal; Est: Estatura; IMC: Índice de Massa Corporal; CC: Circunferência de Cintura; CQ: Circunferência de Quadril; RCQ: Relação Cintura/Quadril; \%GC: Porcentagem de Gordura Corporal; MM: Massa Magra; MG: Massa Gorda; AMBc: Área Muscular de Braço corrigida; FIVM: Força Muscular Máxima Isométrica; DPAP: Desvio-Padrão Anteroposterior; DPML: Desvio-Padrão Médio-lateral; DOT: Deslocamento da Oscilação Total; AmpAP: Amplitude Anteroposterior; AmpML: Amplitude Médio-lateral; VelAP: Velocidade Média Anteroposterior; VelML: Velocidade Média Médio-lateral; FMAP: Frequência Média Anteroposterior; FMML: Frequência Média Médio-lateral.

Tabela 3. Coeficientes de correlação das variáveis explicativas e variáveis estabilométricas na condição olhos fechados das voluntárias. Maceió (AL), 2012.

\begin{tabular}{|c|c|c|c|c|c|c|c|c|c|c|c|c|}
\hline Variável & Idade & $M C$ & Est & IMC & $C C$ & $\mathrm{CQ}$ & $\mathrm{RCQ}$ & $\% G C$ & $\mathrm{MM}$ & MG & $\mathrm{AMBC}$ & FIVM \\
\hline DPAP & $0,06^{*}$ & $0,28^{* *}$ & $0,25^{* *}$ & 0,16 & $0,25^{* *}$ & $0,23^{*}$ & 0,12 & 0,06 & $0,34^{* *}$ & $0,21^{*}$ & 0,14 & $-0,00$ \\
\hline DPML & $0,19^{*}$ & $0,21^{*}$ & 0,11 & 0,17 & $0,26^{* *}$ & $0,25^{* *}$ & 0,07 & 0,14 & $0,19^{*}$ & 0,18 & 0,18 & $-0,07$ \\
\hline DOT & $0,23^{*}$ & 0,08 & 0,09 & 0,07 & 0,15 & $0,19^{*}$ & $-0,01$ & 0,16 & $-0,01$ & 0,11 & 0,04 & $-0,00$ \\
\hline AmpAP & 0,06 & $0,31^{* *}$ & $0,22^{*}$ & $0,21^{*}$ & $0,31^{* *}$ & $0,27^{* *}$ & 0,12 & 0,13 & $0,32^{* *}$ & $0,25^{* *}$ & 0,15 & $-0,01$ \\
\hline AmpML & 0,18 & $0,23^{*}$ & 0,11 & $0,20^{*}$ & $0,27^{* *}$ & $0,26^{* *}$ & 0,09 & 0,17 & $0,20^{*}$ & $0,21^{*}$ & 0,16 & $-0,00$ \\
\hline AEE & 0,14 & $0,28^{* *}$ & $0,19^{*}$ & $0,20^{*}$ & $0,29^{* *}$ & $0,27^{* *}$ & 0,11 & 0,13 & $0,28^{* *}$ & $0,23^{*}$ & $0,19^{*}$ & $-0,06$ \\
\hline VelAP & 0,18 & 0,14 & 0,08 & 0,14 & $0,22^{*}$ & $0,27^{* *}$ & 0,01 & $0,25^{* *}$ & 0,01 & $0,19^{*}$ & 0,05 & 0,00 \\
\hline VelML & $0,25^{* *}$ & $-0,07$ & 0,02 & $-0,05$ & 0,02 & 0,01 & 0,01 & $-0,01$ & $-0,10$ & $-0,03$ & $-0,05$ & $-0,05$ \\
\hline FMAP & 0,10 & $-0,01$ & $-0,08$ & 0,05 & 0,06 & 0,10 & $-0,03$ & $0,21^{*}$ & $-0,18$ & 0,07 & $-0,01$ & 0,06 \\
\hline FMML & 0,04 & $-0,23^{*}$ & $-0,15$ & $-0,16$ & $-0,21^{*}$ & $-0,20^{*}$ & $-0,09$ & $-0,06$ & $-0,28^{* *}$ & $-0,17$ & $-0,18$ & 0,07 \\
\hline
\end{tabular}

Nota: *0,05> p>0,01; ${ }^{* *} p<0,01$; Os valores apresentados são coeficientes de correlação Spearman.

MC: Massa Corporal; Est: Estatura; IMC: Índice de Massa Corporal; CC: Circunferência de Cintura; CQ: Circunferência de Quadril; RCQ: Relação Cintura/Quadril; \%GC: Porcentagem de Gordura Corporal; MM: Massa Magra; MG: Massa Gorda; AMBC: Área Muscular de Braço Corrigida; FIVM: Força Muscular Máxima Isométrica de Extensão do Joelho; DPAP: Desvio-Padrão Anteroposterior; DPML: Desvio-Padrão Médio-lateral; DOT: Deslocamento da oscilação total; AmpAP: Amplitude Anteroposterior; AmpML: Amplitude Médio-lateral; VelAP: Velocidade Média Anteroposterior; VelML: Velocidade Média Médio-lateral; FMAP: Frequência Média Anteroposterior; FMML: Frequência Média Médio-lateral. 
A análise de regressão múltipla (forward) foi realizada para cada variável estabilométrica separadamente e disposta nas Tabelas 4 e 5 em ordem decrescente para valores de $r^{2}$ ajustados nas duas condições experimentais: olhos abertos e fechados.

Tabela 4. Resumo dos modelos de regressão múltipla (forward), com exame do efeito das variáveis explicativas sobre as variáveis estabilométricas na condição de olhos abertos das voluntárias. Maceió (AL), 2012.

\begin{tabular}{|c|c|c|c|c|c|c|c|c|}
\hline Variável & $\beta$ & $t$ & IC95\% & $r$ & $r^{2}$ & $r^{2}$ aj & EPE & E \\
\hline \multicolumn{9}{|l|}{$D P M L$} \\
\hline CC & 0,349 & $3,893^{* *}$ & 0,001 a 0,004 & 0,297 & 0,088 & 0,080 & 0,082 & $10,246^{* *}$ \\
\hline FIVM & $-0,31$ & $-3,463^{* *}$ & $-0,007$ a $-0,002$ & 0,426 & 0,182 & 0,166 & 0,078 & $11,649^{* *}$ \\
\hline$(C C+F I V M)$ & & & & 0,426 & 0,182 & 0,166 & 0,078 & $11,649^{* *}$ \\
\hline \multicolumn{9}{|l|}{ AmpML } \\
\hline$C C$ & 0,376 & $4,193^{*}$ & 0,004 a 0,010 & 0,332 & 0,110 & 0,102 & 0,195 & $13,142^{* *}$ \\
\hline FIVM & $-0,264$ & $-2,944^{* *}$ & $-0,016$ a $-0,003$ & 0,422 & 0,178 & 0,163 & 0,188 & $11,381^{* *}$ \\
\hline$(C C+F I V M)$ & & & & 0,422 & 0,178 & 0,163 & 0,188 & $11,381^{* *}$ \\
\hline \multicolumn{9}{|l|}{$A E E$} \\
\hline$C C$ & 0,206 & $2,014^{*}$ & 0,000 a 0,014 & 0,266 & 0,071 & 0,062 & 0,385 & $8,049^{* *}$ \\
\hline FIVM & $-0,331$ & $-3,486^{* *}$ & $-0,036$ a $-0,010$ & 0,373 & 0,139 & 0,123 & 0,372 & $8,506^{* *}$ \\
\hline $\mathrm{Mm}$ & 0,235 & $2,185^{*}$ & 0,074 a 1,525 & 0,421 & 0,177 & 0,153 & 0,337 & $7,466^{* *}$ \\
\hline$(C C+F I V M+M m)$ & & & & 0,421 & 0,177 & 0,153 & 0,337 & $7,466^{* *}$ \\
\hline \multicolumn{9}{|l|}{ FMML } \\
\hline FIVM & 0,321 & $3,488^{* *}$ & 0,002 a 0,007 & 0,321 & 0,103 & 0,095 & 0,078 & $12,168^{* *}$ \\
\hline (FIVM) & & & & 0,321 & 0,103 & 0,095 & 0,078 & $12,168^{* *}$ \\
\hline \multicolumn{9}{|l|}{ VelAP } \\
\hline$\%$ GC & 0,229 & $2,475^{*}$ & 0,001 a 0,008 & 0,235 & 0,055 & 0,046 & 0,118 & $6,196^{* *}$ \\
\hline Idade & 0,216 & $2,339^{*}$ & 0,045 a 0,547 & 0,319 & 0,102 & 0,085 & 0,116 & $5,965^{* *}$ \\
\hline (\% GC + Idade) & & & & 0,319 & 0,102 & 0,085 & 0,116 & $5,965^{* *}$ \\
\hline \multicolumn{9}{|l|}{ DOT } \\
\hline Idade & 0,257 & $2,73^{* *}$ & 0,171 a 1,07 & 0,257 & 0,066 & 0,057 & 0,209 & $7,480^{* *}$ \\
\hline (Idade) & & & & 0,257 & 0,066 & 0,057 & 0,209 & $7,480^{* *}$ \\
\hline \multicolumn{9}{|l|}{ AmpAP } \\
\hline CC & 0,223 & $2,350^{*}$ & 0,001 a 0,009 & 0,223 & 0,05 & 0,041 & 0,256 & $5,523^{*}$ \\
\hline (CC) & & & & 0,223 & 0,05 & 0,041 & 0,256 & $5,523^{*}$ \\
\hline \multicolumn{9}{|l|}{ FMAP } \\
\hline MM & $-0,199$ & $-2,09^{*}$ & $-0,413$ a $-0,011$ & 0,199 & 0,04 & 0,031 & 0,122 & $4,368^{*}$ \\
\hline (MM) & & & & 0,199 & 0,04 & 0,031 & 0,122 & $4,368^{*}$ \\
\hline \multicolumn{9}{|l|}{ DPAP } \\
\hline RCQ & 0,197 & $2,067^{*}$ & 0,014 a 0,648 & 0,197 & 0,039 & 0,03 & 0,083 & $4,274^{*}$ \\
\hline (RCQ) & & & & 0,197 & 0,039 & 0,03 & 0,083 & $4,274^{*}$ \\
\hline \multicolumn{9}{|l|}{ VelML } \\
\hline Idade & 0,193 & $2,029^{*}$ & 0,010 a 0,842 & 0,193 & 0,037 & 0,028 & 0,193 & $4,118^{*}$ \\
\hline (Idade) & & & & 0,193 & 0,037 & 0,028 & 0,193 & $4,118^{*}$ \\
\hline
\end{tabular}

Nota: ${ }^{*} 0,05<p<0,01 ;{ }^{* *} p<0,01$.

$\beta$ : Coeficiente padronizado de regressão da variável explicativa; $t$ : Teste $t$ pareado; IC95\%: Intervalo de Confiança de $95 \%$; $r$ : Coeficiente de correlação de Pearson; $r^{2}$ : Coeficiente de determinação; $r^{2}$ aj: Coeficiente de determinação ajustado; EPE: Erro-Padrão de Estimativa; $F$ : Teste F; DPML: Desvio-Padrão Médio-lateral; AmpML: Amplitude Médio-lateral; AEE: Área Elíptica da Superfície; FMML: Frequência Média Médio-lateral; VelAP: Velocidade Média Anteroposterior; DOT: Deslocamento da Oscilação Total; AmpAP: Amplitude Anteroposterior; FMAP: Frequência Média Anteroposterior; DPAP: Desvio-Padrão Anteroposterior; VelML: Velocidade Média Médio-lateral; CC: Circunferência de Cintura; RCQ: Relação Cintura/Quadril; FIVM: Força Isométrica Voluntária Máxima; MM: Massa Magra. 
Tabela 5. Resumo dos modelos de regressão múltipla (forward) com exame do efeito das variáveis explicativas sobre as variáveis estabilométricas na condição de olhos fechados das voluntárias. Maceió (AL), 2012.

\begin{tabular}{|c|c|c|c|c|c|c|c|c|}
\hline Variável & $\beta$ & $t$ & IC95\% & $r$ & $r^{2}$ & $r^{2}$ aj & EPE & E \\
\hline \multicolumn{9}{|l|}{ AmpAP } \\
\hline $\begin{array}{l}C C \\
(C C)\end{array}$ & 0,319 & $3,464^{*}$ & $-0,004$ a 0,013 & $\begin{array}{l}0,319 \\
0,319\end{array}$ & $\begin{array}{l}0,102 \\
0,102\end{array}$ & $\begin{array}{l}0,093 \\
0,093\end{array}$ & $\begin{array}{l}0,292 \\
0,292\end{array}$ & $\begin{array}{l}11,996^{* *} \\
11,996^{* *}\end{array}$ \\
\hline \multicolumn{9}{|l|}{$F M M L$} \\
\hline $\begin{array}{l}\text { MM } \\
\text { FIVM } \\
(\mathrm{Mm}+\mathrm{FIVM})\end{array}$ & $\begin{array}{r}-0,326 \\
0,243\end{array}$ & $\begin{array}{l}-3,319^{* *} \\
2,471^{*}\end{array}$ & $\begin{array}{r}-0,391 \text { a }-0,099 \\
0,001 \text { a } 0,007\end{array}$ & $\begin{array}{l}0,241 \\
0,331 \\
0,331\end{array}$ & $\begin{array}{l}0,058 \\
0,110 \\
0,110\end{array}$ & $\begin{array}{l}0,049 \\
0,093 \\
0,093\end{array}$ & $\begin{array}{l}0,085 \\
0,083 \\
0,083\end{array}$ & $\begin{array}{l}6,538^{*} \\
6,479^{* *} \\
6,479^{* *}\end{array}$ \\
\hline \multicolumn{9}{|l|}{ DOT } \\
\hline $\begin{array}{l}\text { CQ } \\
\text { Idade } \\
(C Q+\text { Idade })\end{array}$ & $\begin{array}{l}0,257 \\
0,218\end{array}$ & $\begin{array}{l}2,784^{* *} \\
2,365^{*}\end{array}$ & $\begin{array}{ll}0,226 \text { a } & 1,344 \\
0,117 \text { a } & 1,327\end{array}$ & $\begin{array}{l}0,249 \\
0,330 \\
0,330\end{array}$ & $\begin{array}{l}0,062 \\
0,109 \\
0,109\end{array}$ & $\begin{array}{l}0,053 \\
0,092 \\
0,092\end{array}$ & $\begin{array}{l}0,286 \\
0,280 \\
0,280\end{array}$ & $\begin{array}{l}6,978^{* *} \\
6,438^{* *} \\
6,438^{* *}\end{array}$ \\
\hline \multicolumn{9}{|l|}{$A E E$} \\
\hline $\begin{array}{l}C C \\
(C C)\end{array}$ & 0,307 & $3,321^{\text {** }}$ & 0,006 a 0,025 & $\begin{array}{l}0,307 \\
0,307\end{array}$ & $\begin{array}{l}0,094 \\
0,094\end{array}$ & $\begin{array}{l}0,086 \\
0,086\end{array}$ & $\begin{array}{l}0,572 \\
0,572\end{array}$ & $\begin{array}{l}11,028^{* *} \\
11,028^{* *}\end{array}$ \\
\hline \multicolumn{9}{|l|}{ VMAP } \\
\hline $\begin{array}{l}\mathrm{CQ} \\
(\mathrm{CQ})\end{array}$ & 0,306 & $3,305^{* *}$ & 0,240 a 0,960 & $\begin{array}{l}0,306 \\
0,306\end{array}$ & $\begin{array}{l}0,093 \\
0,093\end{array}$ & $\begin{array}{l}0,085 \\
0,085\end{array}$ & $\begin{array}{l}0,180 \\
0,180\end{array}$ & $\begin{array}{l}10,923^{* *} \\
10,923^{* *}\end{array}$ \\
\hline \multicolumn{9}{|l|}{$D P M L$} \\
\hline $\begin{array}{l}\mathrm{CQ} \\
(\mathrm{CQ})\end{array}$ & 0,302 & $3,260^{* *}$ & 0,120 a 0,494 & $\begin{array}{l}0,302 \\
0,302\end{array}$ & $\begin{array}{l}0,091 \\
0,091\end{array}$ & $\begin{array}{l}0,083 \\
0,083\end{array}$ & $\begin{array}{l}0,093 \\
0,093\end{array}$ & $\begin{array}{l}10,628^{* *} \\
10,628^{* *}\end{array}$ \\
\hline \multicolumn{9}{|l|}{ AmpML } \\
\hline $\begin{array}{l}C C \\
(C C)\end{array}$ & 0,301 & $3,249^{* *}$ & 0,003 a 0,014 & $\begin{array}{l}0,301 \\
0,301\end{array}$ & $\begin{array}{l}0,091 \\
0,091\end{array}$ & $\begin{array}{l}0,082 \\
0,082\end{array}$ & $\begin{array}{l}0,311 \\
0,311\end{array}$ & $\begin{array}{l}10,553^{* *} \\
10,553^{* *}\end{array}$ \\
\hline \multicolumn{9}{|l|}{ DPAP } \\
\hline $\begin{array}{l}C C \\
(C C)\end{array}$ & 0,25 & $2,655^{* *}$ & 0,001 a 0,004 & $\begin{array}{l}0,250 \\
0,250\end{array}$ & $\begin{array}{l}0,062 \\
0,062\end{array}$ & $\begin{array}{l}0,054 \\
0,054\end{array}$ & $\begin{array}{l}0,108 \\
0,108\end{array}$ & $\begin{array}{l}7,051^{\text {** }} \\
7,051^{\text {** }}\end{array}$ \\
\hline \multicolumn{9}{|l|}{$V M M L$} \\
\hline $\begin{array}{l}\text { Idade } \\
\text { (Idade) }\end{array}$ & 0,219 & $2,309^{*}$ & 0,050 a 0,660 & $\begin{array}{l}0,219 \\
0,219\end{array}$ & $\begin{array}{l}0,048 \\
0,048\end{array}$ & $\begin{array}{l}0,039 \\
0,039\end{array}$ & $\begin{array}{l}0,141 \\
0,141\end{array}$ & $\begin{array}{l}5,334^{*} \\
5,334^{*}\end{array}$ \\
\hline \multicolumn{9}{|l|}{ FMAP } \\
\hline $\begin{array}{l}\% G C \\
(\% G C)\end{array}$ & 0,205 & $2,157^{*}$ & 0,000 a 0,006 & $\begin{array}{l}0,205 \\
0,205\end{array}$ & $\begin{array}{l}0,042 \\
0,042\end{array}$ & $\begin{array}{l}0,033 \\
0,033\end{array}$ & $\begin{array}{l}0,0912 \\
0,0912\end{array}$ & $\begin{array}{l}4,652^{*} \\
4,652^{*}\end{array}$ \\
\hline
\end{tabular}

Nota: ${ }^{*} 0,05<p<0,01 ;{ }^{* *} p<0,01$.

$\beta$ : Coeficiente padronizado de regressão da variável explicativa; $t$ : Teste $t$ pareado; IC95\%: Intervalo de Confiança de $95 \%$; $r$ : Coeficiente de correlação de Pearson; $r^{2}$ : Coeficiente de determinação; $r^{2}$ aj: Coeficiente de determinação ajustado; EPE: Erro-Padrão de Estimativa; F: Teste F; AmpAP: Amplitude Anteroposterior; FMML: Frequência Média Médio-lateral; DOT: Deslocamento da Oscilação Total; AEE: Área Elíptica da Superfície; VMAP: Velocidade Média Anteroposterior; DPML: Desvio-Padrão Médio-lateral; AmpML: Amplitude Médio-lateral; DPAP: Desvio-Padrão Anteroposterior; VMML: Velocidade Média Médio-lateral; FMAP: Frequência Média Anteroposterior; \%GC: Percentual de Gordura Corporal.; CC: Circunferência de Cintura; CQ: Circunferência de Quadril.

\section{I S C U S S Ã O}

O objetivo do presente estudo exploratório foi investigar a associação entre diferentes medidas estabilométricas, três medidas indicadoras do estado nutricional - IMC, percentual de gordura corporal e área muscular de braço corrigida -, tipo de distribuição de gordura corporal e força muscular em uma amostra de idosas. Adicionalmente, verificou-se a contribuição relativa das variáveis antropométricas e da força muscular para o desempenho de diferentes variáveis estabilométricas. 
Nos resultados, foi observado que, com exceção da estatura, a razão cintura/quadril (condição olhos abertos), a FIVM (condição olhos fechados) e as variáveis explicativas incluídas no presente estudo apresentaram associações estatisticamente significantes com uma ou mais variáveis estabilométricas. A análise de regressão múltipla (forward) permitiu identificar que as variáveis estabilométricas desvio-padrão médio-lateral (condição olhos abertos) e amplitude anteroposterior (condição olhos fechados) foram as mais influenciadas pelas variáveis explicativas.

Corroborando o descrito na literatura ${ }^{10}$, neste estudo, também foram notáveis as alterações do estado nutricional verificadas na população idosa, sobretudo no que se refere às elevadas médias observadas no IMC $(28,34 \pm 5,02)$ e na percentual de gordura corporal $(40,82 \pm 6,17)$. Levando-se em consideração tais achados, a literatura indica a possibilidade de o excesso de peso e o tipo de distribuição da gordura corporal influenciarem o controle postural dos indivíduos $^{14,30}$

Há pelo menos duas possíveis razões (uma mecânica e outra funcional) que poderiam explicar o fato de a estabilidade postural ser associada às mudanças da composição corporal, incluindo aumento da massa corporal, gordura corporal e modificações da distribuição da gordura corporal. A primeira está relacionada à contribuição dos mecanorreceptores do pé e a sensibilidade cutânea plantar para o controle postural.

O poder discriminatório dos mecanorreceptores pode ser afetado por um estímulo constante e repetitivo, conforme sugerem Hue et al. ${ }^{30}$, os quais atestam que o excesso de peso pode ser um provável candidato para reduzir a sensibilidade de mecanorreceptores. Quando comparados aos não obesos, pessoas obesas geralmente apresentam maiores áreas de contato plantar e maiores valores de pressão média para a maioria dos pontos anatômicos testados ${ }^{31}$, bem como aumento significativo na pressão sob o calcanhar, mediopé e cabeças metatarsais ${ }^{32}$.
Em consequência, os maiores valores de pressão e de áreas de contato nas pessoas obesas podem reduzir a qualidade e/ou a quantidade de informações sensoriais provenientes dos mecanorreceptores plantares. Na verdade, esse fato é sugerido por Teasdale et al..$^{33}$, que demonstraram, em intervenção, que a perda de peso em pacientes obesos e obesos mórbidos proporcionou uma melhora significativa da estabilidade postural (diminuição da amplitude e velocidade do centro de pressão). Ressalta-se ainda que, em idosos, a sensibilidade cutânea plantar e a aferência mecanorreceptora são prejudicadas tanto pelo processo de envelhecimento em si, quanto pela presença de patologias que influenciam negativamente o sistema nervoso periférico ${ }^{34}$.

A segunda provável razão para explicar o motivo da estabilidade postural pode ser as alterações verificadas na circunferência de cintura, que inclui o aumento da massa corporal, gordura corporal e modificações da distribuição da gordura corporal: o modelo de pêndulo invertido. Em outras palavras, quando em pé, o corpo humano é muitas vezes comparado a um pêndulo invertido, girando em torno da articulação do tornozelo ${ }^{11}$. Tem-se sugerido que a obesidade e/ou o sobrepeso, em associação com a distribuição centrípeta da gordura corporal, alterem o equilíbrio, o que modificaria a localização do centro de massa, levando à instabilidade biomecânica do controle postural12,13,30,33.

Portanto, quando uma pessoa obesa, sobretudo idosa, é submetida a uma pequena oscilação para frente, a distribuição anormal de gordura corporal na região abdominal (posição do centro de massa comum relativa ao tornozelo) cede a um torque maior reestabilizante do tornozelo, necessário para recuperar o equilíbrio. Isso sugere que, quando submetidos a um estresse postural diário e outras perturbações, as pessoas obesas, particularmente aqueles com distribuição excessiva de gordura na região abdominal, podem estar em maior risco de cair quando comparadas a indivíduos com peso normal, visto que os indivíduos eutróficos possuem uma maior capacidade 
de gerar torque rápido no tornozelo para recuperar o equilíbrio ${ }^{11,30,33}$.

No presente estudo, observou-se que a massa corporal, IMC, percentual de gordura corporal e a massa gorda, bem como a razão cintura/ quadril, circunferência de cintura e quadril associaram-se significativamente a alterações das variáveis estabilométricas (Tabelas 2 e 3).

Apesar de tanto a percentual de gordura corporal, quanto o IMC, a massa corporal e a massa magra, sozinhas, influenciarem a estabilidade postural, parece que há uma maior contribuição da distribuição da gordura corporal do que o excesso de peso ou adiposidade. Quando analisados os modelos de regressão, pode-se observar que a influência das circunferências de cintura e quadril, ou seja, a distribuição de gordura corporal foi claramente marcante.

Para as variáveis desvio-padrão médio-lateral e amplitude médio-lateral (condição olhos abertos), as quais obtiveram maiores valores de $r^{2}$ (cerca 18,0\% cada) nos modelos finais de regressão, a circunferência de cintura foi determinante para explicar a variação da estabilidade postural, e nem a massa corporal, percentual de gordura corporal, massa gorda e IMC foram incluídas nos modelos.

A circunferência de cintura foi responsável por 8,8\% da variação do desvio-padrão médio-lateral e $11,0 \%$ da variação da amplitude médio-lateral. Vale salientar que há evidências crescentes de que a instabilidade médio-lateral é um importante marcador estabilométrico de compro-metimento do equilíbrio funcional ${ }^{35}$. Já no modelo final de regressão para a variável amplitude anteroposterior (condição olhos fechados), a circun-ferência de cintura, sozinha, foi responsável por cerca de 10,0\% da variação.

Nos demais modelos, a influência da circunferência de cintura variou de 5,0 a 6,8\%, e a razão cintura/quadril foi incluída em um modelo (desvio-padrão anteroposterior) contribuindo com 3,0\%. Contudo, o IMC, a massa corporal e a massa gorda não foram inseridos em nenhum modelo de regressão, e a percentual de gordura corporal em apenas um modelo, contribuindo apenas com $4,7 \%$ da variação da variável velocidade anteroposterior (Tabela 4).

Na condição olhos fechados, as circunferências de cintura e de quadril prevaleceram nos modelos de regressão. Essas variáveis foram incluídas em $60 \%$ de todos os modelos, e, em seis deles, foram as únicas variáveis explicativas presentes (Tabela 5).

No presente estudo, identificou-se uma elevada colinearidade entre as variáveis de massa corporal, IMC, percentual de gordura corporal, massa gorda, relação de cintura/quadril, circunferência de cintura e circunferência de quadril; sendo assim, foram incluídas nos modelos de regressão múltipla apenas as variáveis que não violaram os pressupostos de inclusão e que se correlacionavam mais fortemente com a variável resposta.

Hue et al. ${ }^{30}$, utilizando como amostra sujeitos com idades entre 24 e 61 anos, atribuíram à massa corporal uma grande relevância para as alterações da estabilidade postural. No estudo citado, para a variável estabilométrica velocidade média, a massa corporal foi responsável por 55\% da variação observada. Vale salientar que, no estudo citado, a inclusão da massa corporal nos modelos de regressão foi forçada, e as medidas que verificavam o estado nutricional (IMC) e a distribuição de gordura corporal, como a relação de cintura/quadril, circunferência de cintura e circunferência de quadril, foram excluídas de suas análises pela elevada colinearidade com a massa corporal, não permitindo atribuir um peso relativo à massa corporal e sua distribuição isoladamente, ou seja, não foi possível verificar se, de fato, a massa corporal era, entre todas as variáveis, a maior responsável pelas grandes variações observadas.

No presente estudo, observou-se, claramente, que as medidas de distribuição de gordura corporal (relação de cintura/quadril, circunferência de cintura e circunferência de quadril) influenciam sobremaneira o controle postural das idosas, visto 
que foram incluídas em $60 \%$ dos modelos de regressão (Tabelas 4 e 5), enquanto a massa corporal, ou mesmo o IMC, não esteve presente em nenhuma. Dessa forma, os achados do presente estudo sugerem que a instabilidade postural em idosos parece ser mais bem explicada, no tocante à composição corporal, pela influência do acúmulo anormal de gordura na região central do corpo, levando a um maior deslocamento do centro de massa no modelo de pêndulo invertido do que pelo excesso de peso em si.

Apesar de observadas fortes evidências de que o aumento da gordura corporal e sua distribuição centrípeta estejam possivelmente relacionadas a maiores alterações do controle postural, pesquisas verificaram que indivíduos classificados em baixo peso ou desnutridos, sobretudo idosos, estão sob maior risco de desequilíbrio e quedas $^{17,18}$.

No presente estudo, não foram encontradas associações negativas e estatisticamente significativas entre as variáveis estabilométricas e o baixo peso, avaliado pelo IMC. Apenas para a variável massa corporal foi estabelecida uma associação: indivíduos com menor massa corporal pareciam aumentar a frequência média de oscilação médio-lateral do centro de pressão na condição olhos fechados, ocasionando maior instabilidade (Tabela 3).

A observação sugere que os indivíduos com baixos valores de massa corporal talvez solicitem maiores ajustes de controle neuromuscular para manter o equilíbrio devido à diminuição de massa muscular associada. Contudo, nos modelos de regressão, nem a massa corporal nem qualquer outra variável explicativa que se correlacione fortemente a ela (IMC, por exemplo) foi incluída de modo a associar-se negativamente à frequência de oscilação do centro de pressão.

Embora o índice de massa corporal seja amplamente utilizado em estudos populacionais por ser uma forma simples, barata e útil, tanto em nível individual quanto populacional, além de permitir comparações com outros estudos (nacionais ou internacionais), expressar as reservas energéticas do indivíduo e apresentar elevada correlação com a massa corporal não destingue adequadamente massa gorda e magra, especialmente massa muscular. Além disso, apresenta sérias restrições quanto ao uso na população idosa ${ }^{36}$. Dessa forma, outras medidas que permitam um diagnóstico do estado nutricional e que possam ser utilizadas sem grandes restrições nessa população são desejadas. No presente estudo, foram incluídas mais duas medidas de estado nutricional: percentual de gordural corporal e área muscular de braço corrigida.

A avaliação da percentual de gordura corporal permite quantificar a massa magra, enquanto a área muscular de braço corrigida permite verificar, indiretamente e de maneira acurada, a massa muscular do idoso ${ }^{25}$. Sabe-se que, com o avançar da idade, há um declínio na massa corporal magra, nomeadamente no que se refere à massa muscular. Tais alterações têm sido descritas como importantes fatores de declínio da funcionalidade e capacidade física, além de elevarem o risco de quedas ${ }^{9,17}$. Entretanto, Visser et al. ${ }^{37}$ não encontraram qualquer associação entre massa muscular e autorrelato de deficiência ou desempenho de membros inferiores.

Nesse sentido, a massa magra pareceu influenciar negativamente apenas a frequência anteroposterior e mediolateral nas situações olhos abertos e fechados, respectivamente (Tabelas $4 \mathrm{e}$ 5). Ao contrário do que sugere a literatura, a área muscular de braço corrigida não pareceu associar-se à alteração de controle postural em idosas uma vez que apresentou correlações fracas e positivas apenas com duas variáveis respostas: desvio-padrão médio-lateral (condição olhos abertos) e área elíptica do estatocinesiograma (condição olhos fechados). Era de se esperar que quanto menor a área muscular de braço corrigida (que exprime a massa muscular), maiores fossem as alterações de equilíbrio ${ }^{38}$.

O idoso normalmente apresenta grande redução da força muscular associada à perda de massa muscular (medida aqui de forma indireta pela área muscular de braço corrigida) ${ }^{21}$. Na presente pesquisa, a medida escolhida para verificar os níveis de força muscular foi a FIVM dos exten- 
sores do joelho, que apresentou, na condição olhos abertos, correlações estatisticamente significativas $(p<0,05)$ com algumas variáveis estabilométricas.

Para as variáveis desvio-padrão médio-lateral, amplitude médio-lateral e área elíptica do estatocinesiograma, as associações verificadas foram fracas e negativas. Ou seja, quanto menor a força muscular, maior a instabilidade postural. Contudo, para a variável frequência média médio-lateral (condição olhos abertos), a correlação foi positiva $(r=0,347 ; p<0,01)$, o que não era esperado.

Embora a perda de massa muscular esteja associada ao declínio da força em idosos, essa diminuição é muito mais rápida do que a perda concomitante de massa muscular, sugerindo um declínio na qualidade do músculo por causa de uma função deteriorada da junção neuromuscular $^{39}$. Além disso, manter ou ganhar massa muscular não impede diminuição da força muscular associada ao envelhecimento ${ }^{40}$.

A idade vem sendo citada em diversos estudos como um importante preditor de quedas e instabilidade postural ${ }^{35}$. Em virtude disso, foi incluída nas análises de correlação e regressão múltipla. A média de idade amostral do presente estudo foi de 70,1 anos, com amplitude entre 60 e 87 anos, e obtiveram-se baixas correlações com as variáveis estabilométricas (Tabelas 2 e 3 ).

Nos modelos de regressão, a idade foi incluída em três modelos na condição olhos abertos (Tabela 4) e em dois modelos na condição olhos fechados (Tabela 5). A maior influência da idade foi verificada na variável estabilométrica, deslocamento da oscilação total (olhos abertos), sendo responsável por $6,6 \%$ da variação total para esse parâmetro. Era esperada uma maior influência da idade sobre as variáveis estabilométricas visto que há um declínio significativo dos sistemas que regulam o controle postural, como o visual, somatossensorial e o vestibular ${ }^{41}$.

Outra possível variável de confundimento incluída nas análises de correlação e regressão múltipla foi a estatura. A estatura, per se, é citada como um fator independente de alterações do controle postura ${ }^{24}$. Em estudo clássico, Era et al. ${ }^{42}$ identificaram que, em idosos, quanto menor a estatura, melhor o desempenho das mulheres nos teste de equilíbrio quando comparadas a indivíduos do sexo oposto. Na presente pesquisa, a estatura associou-se significativamente com as variáveis estabilométricas apenas na condição olhos fechados (Tabela 3), corroborando os achados de Era et al. ${ }^{42}$, contudo ela não foi incluída nos modelos finais de regressão.

Os pontos fortes do presente estudo primariamente foram a homogeneidade, o tamanho e o perfil da amostra. Ademais, até o término do presente estudo, não havia publicações relevantes que buscaram verificar a influência da composição corporal, distribuição da gordura corporal e força muscular no controle postural avaliado por plataforma de força. Observa-se também que estudos semelhantes, mas com uma amostra distinta (crianças, por exemplo), sempre utilizaram correlações simples apenas, e não modelos de regressão com o intuito de verificar a contribuição relativa das variáveis de estado nutricional, distribuição de gordura corporal e força muscular sobre as variáveis estabilométricas de idosas.

Algumas limitações do estudo merecem ser discutidas: 1) a estabilometria, sozinha, talvez não seja a melhor forma de identificar instabilidade postural. Apesar de poder-se quantificar as oscilações posturais, condições que imprimam desequilíbrio são desejadas; 2) outros métodos complementares, como registros eletromiográficos e baropodométricos, poderiam fornecer maiores informações. Este estudo investiga uma área limitada dos mecanismos fisiológicos envolvidos no controle da postura humana, sendo a compreensão de toda a dinâmica relacionada ao controle postural ainda um campo aberto de pesquisa; 3) o fato de a amostra ter sido não probabilística pôde ter causado viés de seleção; 4) é provável que o uso de um teste sensorial plantar possa fornecer informações sobre a neuropatia pré-clínica, especialmente em indivíduos idosos e obesos com índice elevado de sensibilidade à insulina. 


\section{O N C L U S Ã O}

Um adequado controle postural é um pré-requisito essencial para as tarefas diárias, servindo de base para a realização da maioria dos movimentos, sobretudo em idosos. Diante do exposto, pode-se concluir que um número relevante de variáveis explicativas estudadas que se referem ao estado nutricional, distribuição de gordura corporal e força muscular associa-se significativamente a uma ou mais variáveis estabilométricas, o que pode contribuir para alterações de estabilidade postural. Adicionalmente, a análise de regressão sugere que, dentre as variáveis explicativas, a distribuição de gordura corporal e a diminuição de força muscular, não o excesso de massa corporal, são responsáveis, em parte, pelas alterações da estabilometria de idosas.

Claramente, estudos clínicos prospectivos devem ser realizados com o intuito de identificar os benefícios das modificações de composição corporal, distribuição da gordura corporal e força muscular no controle postural e, consequentemente, no risco de quedas em idosos.

\section{COLABORADORES}

FMS SIQUEIRA colaborou na concepção, desenho, coleta, análise, interpretação dos dados do estudo e elaboração do manuscrito. AAR GERALDES colaborou na concepção do estudo e revisão do manuscrito.

\section{REFERÊ NCIAS}

1. Kirkwood TBL, Austad SN. Why do we age? Nature. 2000; 408(6809):233-8. http://dx.doi.org/10.1038/ 35041682

2. Rubenstein LZ. Falls in older people: Epidemiology, risk factors and strategies for prevention. Age Ageing. 2006; 35(2):37-41. http://dx.doi.org/10.10 93/ageing/afl084

3. Mancini M, Horak FB. The relevance of clinical balance assessment tools to differentiate balance deficits. Eur J Phys Rehabl Med. 2010; 46(2):239-48.

4. Duarte M, Freitas SMSF. Revisão sobre posturografia ba-seada em plataforma de força para avaliação do equilíbrio. Rev Bras Fisio. 2010; 14(3):183-92.

5. Abrahamová D, Hlavacka F. Age-related changes of human balance during quiet stance. Physiol Res. 2008; 57(6):957-64.
6. Nardone A, Schieppati M. The role of instrumental assessment of balance in clinical decision making. Eur J Phys Rehabil Med. 2010; 46(2):221-37.

7. Pajala S, Era P, Koskenvuo M, Kaprio J. Force platform balance measures as predictors of indoor and outdoor falls in community-dwelling women aged 63-76 years. J Gerontol. 2008; 63(2):171-8.

8. Lihavainen $K$, Sipilä S, Rantanen T, Sihvonen S, Sulkava R, Hartikainen S. Contribution of musculoskeletal pain to postural balance in community-dwelling people aged 75 years and older. J Gerontol. 2010; 65(9):990-6. http://dx.doi. org/10.1093/gerona/glq052

9. Baumgartner RN, Koehler KM, Gallagher D, Romero L, Heymstleld SB, Ross RR, et al. Epidemiology of sarcopenia among the elderly in New Mexico. Am J Epidemiol. 1998; 147(8):755-63.

10. Waters DL, Hale L, Grant AM, Herbison P, Goulding A. Osteoporosis and gait and balance disturbances in older sarcopenic obese New Zealanders. Osteoporos Int. 2010; 21(2):351-7. http://dx.doi. org/10.1007/s00198-009-0947-5

11. Corbeil P, Simoneau M, Rancourt D, Tremblay A, Teasdale N. Increased risk for falling associated with obesity: Mathematical modeling of postural control. IEEE Trans Neural Syst Rehabil Eng. 2001; 9(2):12636. http://dx.doi.org/10.1109/7333.928572

12. Blaszczyk JW, Plewa M, Cies J, Zahorska-Markiewicz B, Markiewicz A. Effects of excessive body weight on postural control. J Biomech. 2009; 42(9):1295-300. http://dx.doi.org/10.1016/j.jbiomech.2009.03.006

13. Mainenti M, Rodrigues EC. Adiposity and postural balance control: Correlations between bioelectrical impedance and stabilometric signal in elderly Brazilian women. Clinics. 2011; 66(9):1513-8. http://dx.doi.org/10.1590/S1807-593220110009 00001

14. Handrigan $G$, Hue $O$, Simoneau $M$, Corbeil $P$, Marceau P, Marceau S, et al. Weight loss and muscular strength affect static balance control. Int J Obesity. 2010; 34(5):936-42. http://dx.doi.org/ 10.1038/ijo.2009.300

15. Winters K. Body composition predicts bone mineral density and balance in premenopausal women. J Women's Health Gender-Based Med. 2000; 9(8):856-72.

16. Hassinen M, Komulainen P, Lakka TA, Väisänen SB, Rauramaa R. Associations of body composition and physical activity with balance and walking ability in the elderly. J Phys Act Health. 2005; 3:298-306.

17. Kinney JM. Nutritional frailty, sarcopenia and falls in the elderly. Curr Opin Clin Nutr Metabol Care. 2004; 7(1):15-20.

18. Miller MD, Thomas JM, Cameron ID, Sheng Chen J, Sambrook PN, March LM, et al. BMI: A simple, rapid 
and clinically meaningful index of under-nutrition in the oldest old? Br J Nutr. 2008; 101(9):1300-5.

19. Young $Y$, Myers AH, Provenzano G. Factors associated with time to first hip fracture. J Aging Health. 2001; 13(4):511-26.

20. Rantanen T, Guralnik JM, Sakari-Rantala R, Leveille S, Simonsick EM, Ling $S$, et al. Disability, physical activity, and muscle strength in older women: The women's health and aging study. Arch Phys Med Rehabil. 1999; 80(2):130-5. http://dx.doi.org/ 10.1016/S0003-9993(99)90109-0

21. Orr R. Contribution of muscle weakness to postural instability in the elderly: A systematic review. Eur Phys Rehebil Med. 2010; 46(2):183-220.

22. Jubrias SA, Odderson IR, Esselman PC, Conley KE. Decline in isokinetic force with age: Muscle crosssectional area and specific force. Eur J Physiol. 1997; 434(3):246-53.

23. Lohman TG, Roche AF, Martorell R. Anthropometric Standardization Reference Manual. Champaign (IL): Human Kinetics Books; 1991.

24. Chiari L, Rocchi L, Cappello A. Stabilometric parameters are affected by anthropometry and foot placement. Clin Biomec. 2002; 17(9-10):666-77.

25. Frisancho AR. New norms of upper limb fat and muscle areas for assessment of nutritional status. Am J Clin Nutr. 1981; 34(11):2540-5.

26. Oliveira DWL, Geraldes AR. Validação de equação preditiva para a composição corporal em idosas alagoanas [mestrado]. Alagoas: Universidade Federal de Alagoas; 2011.

27. Edwards RH, Young A, Hosking GP, Jones DA. Human skeletal muscle function: Description of tests and normal values. Clin Sci Mol Med. 1977; 52(3):283-90.

28. Lafond D, Corriveau H, Hebert R, Prince F. Intrasession reliability of center of pressure measures of postural steadiness in healthy elderly people. Arch Phys Med Rehabil. 2004; 85(6):896-901. http://dx.doi.org/10.1016/j.apmr.2003.08.089

29. Riley MA, Baker AA, Schmit JM, Weaver E. Effects of visual and auditory short-term memory tasks on the spatiotemporal dynamics and variability of postural sway. J Motor Behav. 2005; 37(4):311-24. http://dx.doi.org/10.3200/JMBR.37.4.311-324

30. Hue O, Simoneau M, Marcotte J, Berrigan F, Doré J, Marceau $\mathrm{P}$, et al. Body weight is a strong predictor of postural stability. Gait Posture. 2007; 26(1):32-8. http://dx.doi.org/10.1016/j.gaitpost.2006.07.005

31. Birtane $M$, Tuna $H$. The evaluation of plantar pressure distribution in obese and non-obese adults. Clin Biomech. 2004; 19(10):1055-9. http:// dx.doi.org/10.1016/j.clinbiomech.2004.07.008
32. Hills AP, Hennig EM, McDonald M, Bar-Or O. Plantar pressure differences between obese and non-obese adults: A biomechanical analysis. Int J Obest Relat Metab Disord. 2001; 25(11):1674-9.

33. Teasdale N, Hue O, Marcotte J, Berrigan F, Simoneau M, Dore J, et al. Reducing weight increases postural stability in obese and morbid obese men. Int J Obest. 2007; 31(1):153-60. http://dx.doi.org/10.10 38/sj.ijo.0803360

34. Mickle KJ, Munro BJ, Lord SR, Menz HB, Steele JR. Foot pain, plantar pressures, and falls in older people: A prospective study. J Am Geriatr Soc. 2010; 58(10):1936-40. http://dx.doi.org/10.1111/j.1532-54 15.2010.03061.x

35. Piirtola M, Era P. Force platform measurements as predictors of falls among older people: A review. Gerontology. 2006; 52(1):1-16. http://dx.doi.org/ 10.1159/000089820

36. Cervi A, Franceschini CC, Priore E, Federal U, Regional U, Grande R, et al. Análise crítica do uso do índice de massa corporal para idosos. Rev Nutr. 2005; 18(6):765-75. http://dx.doi.org/10.1590/ S1415-52732005000600007

37. Visser M, Langlois J, Guralnik JM, Cauley J, Kronmal $R$, Robbins J, et al. High body fatness, but not low fat-free mass, predicts disability in older men and women: The cardiovascular health study. Am J Clin Nutr. 1998; 68(3):584-90.

38. Broadwin J, Goodman-Gruen D, Slymen D. Ability of fat and fat-free mass percentages to predict functional disability in older men and women. J Am Geriatr Soc. 2001; 49(12):1641-5. http://dx. doi.org/10.1111/j.1532-5415.2001.49273.x

39. Goodpaster B, Park S. The loss of skeletal muscle strength, mass, and quality in older adults: The health, aging and body composition study. J Gerontol. 2006; 61(10):1059-64.

40. Morley JE, Baumgartner RN, Roubenoff R, Mayer J, Nair KS. Sarcopenia. J Lab Clin Med. 2001; 137(4):231-43.

41. Horak FB. Postural orientation and equilibrium: What do we need to know about neural control of balance to prevent falls? Age Ageing. 2006; 35(2):7-11. http://dx.doi.org/10.1093/ageing/ afl077

42. Era P, Schroll M, Ytting H, Gause-Nilsson I, Heikkinen E, Steen B. Postural balance and its sensory-motor correlates in 75-year -old men and women: A cross-national comparative study. J Gerontol.1996; 51(2):M53-63. http://dx.doi.org/ 10.1093/gerona/51A.2.M53 